\title{
Growth hormone deficiency and phenotypic features in four cases of 22q11.2 deletion syndrome
}

Dalvir Kular¹, Joanne Baker², Mehul Dattani ${ }^{1}$

${ }^{1}$ Genetics and Epigenetics in Health and Disease Section, Genetics and Genomic Medicine Programme, UCL Institute of Child Health, London WC1N 1EH, UK

${ }^{2}$ Department of Paediatrics, Canterbury Hospital, Canterbury, Kent, CT1 3NG, UK

\section{Background}

- 22q11.2 deletion syndrome (22q11DS) displays a wide phenotypic spectrum.

- It is the most common deletion syndrome with an estimated incidence of 1 in 4000 children 1 .

- Short stature is a phenotypic feature of the spectrum.

- Uncommonly, growth hormone deficiency (GHD) has been identified as a cause of short stature within this population².

We present a case series of four 22q11DS patients with concurrent GHD that have been followed up in our paediatric endocrinology clinics.

\section{Case Series}

All four patients were referred to clinic for short stature with heights below the 0.4 th centile. They subsequently underwent GH provocation testing (Table 1) and were diagnosed with GHD. No other pituitary hormone deficiencies were identified and coeliac screen was negative in all four patients.

\section{Patient 1 (Older brother of Patient 2)}

Male patient, born at 37 weeks gestation by emergency caesarean section to non-consanguineos parents. Birth weight $2.72 \mathrm{~kg}$. Background of left hemiparesis secondary to right polymicrogyria, global developmental delay and congenital heart disease. Inherited 22q11.2 deletion from father. Midparentile centile $=9$ th with range $<0.4$ th to $50^{\text {th }}$. Dysmorphic features consistent with 22q11.2 deletion. Bone age reported as 5-6 years at chronological age 8.3 years. Started on daily recombinant human growth hormone ( $\mathrm{r}-\mathrm{hGH})$ at age 4.5 years. Both Patients 1 and 2 had a period of non-compliance to treatment.

\section{Patient 2 (Younger brother of Patient 1)}

- Male patient, born prematurely at 34 weeks gestation, labour was induced for polyhydramnios. Birth weight of $2.47 \mathrm{~kg}$. Background of left hemiparesis secondary to right polymicrogyria, autism, global developmental delay and congenital heart disease. Dysmorphic features consistent with 22q11.2 deletion. Started on daily r-hGH at age 5 years.

\section{Patient 3}

- Male patient, born premature at 33+4 weeks gestation by emergency caesarean section to non-consanguineos parents. Birth weight $2.2 \mathrm{~kg}$ Hypocalcaemic at birth. Background of cryptorchidism of left testis requiring orchidopexy, complex congenital heart disease, subcutaenous cleft palate, severe GORD and left vocal cord palsy. Both parents did not have 22q11.1 deletion on genetic testing, although paternal cousin was found to have 22q11DS. Dysmorphic features consistent with 22q11.2 deletion. Feeding difficulties requiring prolonged $\mathrm{NG}$ feeding. Started on daily $\mathrm{r}-\mathrm{hGH}$ at age 3.3 years.

\section{Patient 4}

Female patient. Birth history unavailable. Birth weight $3.18 \mathrm{~kg}$. Nonconsanguineous parents. Mid-parental height $160.5 \mathrm{~cm}$. Background of complex congenital heart disease. 22q11.2 deletion inherited from mother. Dysmorphic features consistent with $22 q 11.2$. Started on daily $\mathrm{r}-\mathrm{hGH}$ at age 8 years

\section{Growth charts of height pre and post r-hGH ${ }^{3}$}

Figure 1. Patient 1

Boys

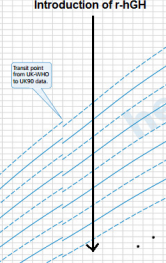

Figure 3. Patient 3

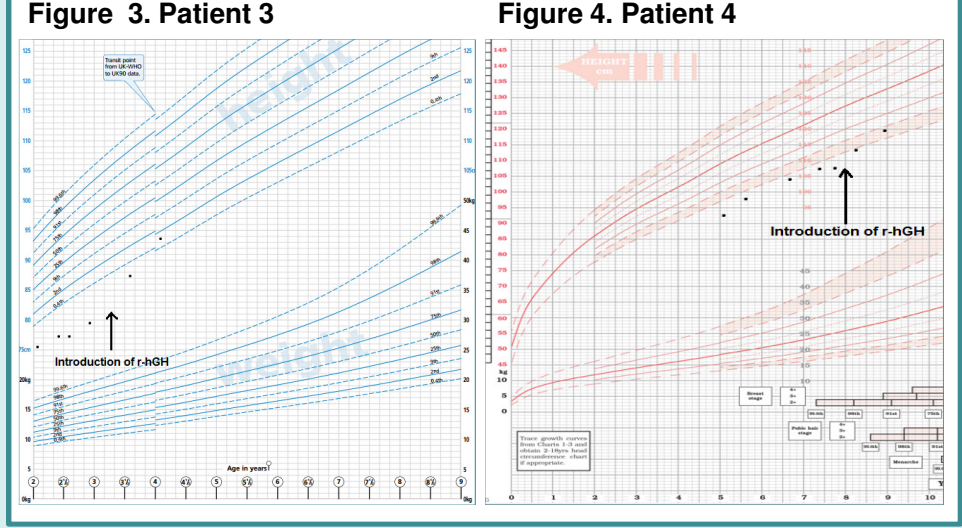

\section{Conclusion}

- The patients were all commenced on GH replacement therapy, with an excellent response (Figures 1-4).

Our data suggest that 22q11DS patients with short stature should be appropriately investigated for GHD if they manifest poor growth.

Early treatment with r-hGH may help optimise height.

\section{References}

1. Botto et al. Pediatrics 2003. 112;101-117.

2. Weinzimer et al. Pediatrics. 1998 May;101(5):929-32

3. Growth charts taken from RCPCH website. http://www.rcpch.ac.uk/childhealth/research-projects/uk-who-growth-charts/uk-growth-chart-resources-2-18years/school-age \& http://www.healthforallchildren.com/shop-base/shop/growthcharts/uk90-four-in-one-charts-duo-decimal/

Table 1. Evaluation for Growth Hormone Deficiency

\begin{tabular}{|c|c|c|c|c|c|c|}
\hline Patient & $\begin{array}{c}\text { Age } \\
\text { (years) }\end{array}$ & MRI Brain and Pituitary appearance & $\begin{array}{c}\text { Source of 22q11 } \\
\text { Deletion }\end{array}$ & $\begin{array}{c}\text { GH peak } \\
\text { (ug/l) }\end{array}$ & IGF-1 & IGFBP-3 \\
\hline 1 & 3 & Right-sided polymicrogyria & Paternal & 5.8 & $7.1 \mathrm{pmol} / \mathrm{l}(4-20)$ & Not tested \\
\hline 2 & 3 & Small anterior pituitary and right-sided polymicrogyria & Paternal & 3.2 & $19 \mathrm{pmol} / \mathrm{I}(4-20)$ & Not tested \\
\hline 3 & 2.7 & Small anterior pituitary, normal posterior pituitary and stalk & Sporadic & 5.0 & $29 \mathrm{ng} / \mathrm{ml}(49-289)$ & $1.55 \mathrm{mg} / \mathrm{L} \mathrm{(0.9-4.3)}$ \\
\hline 4 & 7.16 & Normal & Maternal & 6.5 & $30 \mathrm{ng} / \mathrm{ml}(45-302)$ & $1.78 \mathrm{mg} / \mathrm{L} \mathrm{(1.6-6.5)}$ \\
\hline
\end{tabular}

